\title{
Existence of phase-locking in the Kuramoto system under mean-field feedback *
}

\author{
A. Franci ${ }^{*}$ W. Pasillas-Lépine ${ }^{* *}$ A. Chaillet ${ }^{* * *}$ \\ *Univ. Paris Sud 11 - L2S - Supélec, Gif sur Yvette, 91192 France \\ (e-mail: franci@lss.supelec.fr) \\ ** CNRS - L2S, same address (e-mail: pasillas@lss.supelec.fr) \\ *** EECI - L2S - Univ. Paris Sud 11, same address (e-mail: \\ chaillet@supelec.fr)
}

\begin{abstract}
Motivated by the development of Deep Brain Stimulation (DBS) for neurological diseases, we study a network of interconnected oscillators under the influence of a proportional mean-field feedback. Under standard assumptions, this system can be reduced to a modified version of the Kuramoto model of coupled nonlinear oscillators. In the first part of the paper we show that, in general, no oscillating phase-locked solution can co-exist with any non-zero feedback gain. In the second part we propose a new characterization of phase-locking between Kuramoto oscillators. In particular we derive a fixed point equation for the Kuramoto system under mean-field feedback and we show how, generically, the "standard" (with zero feedback gain) Kuramoto fixed point equation is locally invertible in terms of the implicit function theorem.
\end{abstract}

Keywords: Kuramoto Oscillators, Mean-field Feedback, Phase-Locking, Deep Brain Stimulation.

\section{INTRODUCTION}

In most automatic control applications, synchronization is a goal to achieve. For instance, formations of autonomous vehicles (Sarlette, 2009; Sepulchre et al., 2007, 2008), consensus protocols (Scardovi et al., 2007; Olfati-Saber and Murray, 2004; Sarlette, 2009) and master-slave control of mechanical systems (Nijmeijer and Rodriguez-Angeles, 2003) can all be formulated as a synchronization objective. For some applications, however, synchronization is an undesired effect and the aim of the control law is then to "desynchronize". One of these applications is that of Deep Brain Stimulation for Parkinson's Disease (PD), which is the motivation of the present article.

Under healthy conditions subthalamic nucleus (STN) neurons fire in an uncorrelated (i.e., desynchronized) manner (Sarma et al., 2010). In PD patients, STN neurons form a cluster of synchronous periodic activity that leads to limb tremor by activating premotor areas and the motor cortex (Alberts et al., 1969; Volkmann et al., 1996). In order to overcome tolerance to pharmaceutical therapies, some patients undergo Deep Brain Stimulation (DBS). Through a pair of implanted electrodes, a low voltage "high"-frequency $(>100 \mathrm{~Hz})$ electrical input is permanently injected in the STN. This leads to a drastic reduction of the physical symptoms (Benabid et al., 1991). At present this electrical signal is periodic and generated by a standard artificial pacemaker (open-loop control) and is consequently not optimized for the purpose. Despite its therapeutic success, little is still known about the exact

\footnotetext{
* An earlier version of this paper has been submitted to the IEEE CDC 2010. We are thankful to the anonymous CDC reviewer who pointed out, by showing a counter-example, an issue that lead us to the definition of oscillating phase-locked solutions. This concept helped us to clarify and simplify the proof of our main result.
}

functioning of DBS (Hammond et al., 2008). For each patient an empirical parameter tuning is needed, which may take up to several days and which is not guaranteed to be effective (Rodriguez-Oroz and et al., 2005). Moreover patients can develop side effects or tolerance to DBS (Kumar et al., 2003) with long term treatment. Also, the permanent electrical stimulation leads to a fast discharge of the pacemaker batteries and, consequently, to further surgical operations to change them.

In order to both provide theoretical justifications to DBS and to bypass the above limitations by exploiting cerebral measurements, we develop a rigorous analysis based on a simplified model. More precisely, we analyze synchronization and desynchronization phenomena in coupled complex Landau-Stuart oscillators subject to a scalar input modeling the effect of DBS. The DBS signal is taken proportional to the mean-field of the neuronal population. Due to heterogeneities in the media, the contribution of each agent to the mean-field is seen as an unknown parameter. In the same way, the influence of the DBS signal on each neuron is modeled as an unknown gain. The coupling topology is also taken to be arbitrary, allowing for a general time-invariant interconnection. This approach thus allows to represent any recording-stimulation setup as well as any coupling topology. Nonetheless, we point out that it does not detail the neuronal dynamics, nor the electrode setup. Under standard assumptions, our model reduces to a modified version of Kuramoto coupled oscillators. This model, originally developed in the seminal works (Kuramoto, 1984; Winfree, 1980), has been already exploited to analyze both synchronization and desynchronization phenomena (Scardovi et al., 2007; Chopra and Spong, 2009; Acebrón et al., 2005; Aeyels and Rogge, 2004; Jadbabaie et al., 2004; Brown et al., 2003; Sepulchre 
et al., 2007; Pikovsky et al., 2001; Strogatz, 2000; Franci et al., 2010). After having formally defined the concept of phase-locked solutions, we show that, for a generic class of interconnections between the oscillators, the existence of perfectly phase-locked oscillating solutions is not compatible with any non-zero mean-field proportional feedback. This analytical result confirms the expectations of a closed-loop desynchronizing strategy. The proof of the main theorem relies on two fundamental steps. Their interest goes beyond the technical aspects of the proof, as they underline intrinsic properties of the system. In the first result we completely characterize the oscillating phase-locked solutions of the Kuramoto system under mean-field feedback through a fixed point equation. In the second result we show that, generically, the "standard" Kuramoto fixed points equation (that is for zero feedback gain) can be locally inverted around any of its solutions through the implicit function theorem. This result extends the results in (Aeyels and Rogge, 2004), where the fixed point equation is solved (i.e. inverted), in the particular case of the all-to-all coupling.

The paper is organized as follows. In Section 2 we derive a simple model of interconnected oscillators under proportional mean-field feedback. In Section 3 we formally define phase-locked solution and show their generic disappearance under mean-field feedback, and give a new characterization of phase-locking between Kuramoto oscillators. Proofs are given in Section 4

\section{MODEL DERIVATION}

A simple representation of the limit cycle behavior that appears in periodically spiking neurons (Izhikevich, 2007, Sections $6.1 .3,6.1 .4$ ) is given by the Landau-Stuart oscillator (Kuramoto, 1984):

$$
\dot{z}=\left(\mathrm{i} \omega_{\circ}+\rho^{2}-|z|^{2}\right) z, \quad z \in \mathbb{C},
$$

which represents a normal form of the Andronov-Hopf bifurcation, where $\omega_{\circ} \in \mathbb{R}$ and $\rho \in \mathbb{R}_{>0}$ denote the natural frequency and the radius of the oscillation, respectively. The model for $N \in \mathbb{N}_{\geq 1}$ diffusively coupled oscillators is then given by

$\dot{z}_{i}=\left(\mathrm{i} \omega_{i}+\rho_{i}^{2}-\left|z_{i}\right|^{2}\right) z_{i}+\sum_{j=1}^{N} \kappa_{i j}\left(z_{j}-z_{i}\right), \quad \forall i=1, \ldots, N$,

where $\kappa_{i j}, i, j=1, \ldots, N$, denotes the coupling gain from oscillator $j$ to oscillator $i$. We denote $\omega:=\left[\omega_{i}\right]_{i=1, \ldots, N} \in$ $\mathbb{R}^{N}$ as the vector of natural frequencies. We consider an arbitrary interconnection topology, and thus allow $\kappa_{i j}$, $i, j=1, \ldots, N$, to be arbitrary in our study. The possibility of considering any interconnection topology is an interesting particularity of the approach presented here with respect to the existing literature.

The presence of a limited number of electrodes and their large size with respect to the neuronal scale, makes the mean-field (i.e. the mean membrane voltage) of the ensemble the only plausible measurement in DBS. The output of our system is therefore

$$
y:=\sum_{j=1}^{N} \alpha_{j} \operatorname{Re}\left(z_{j}\right),
$$

which is referred to as the mean-field of the ensemble, where $\alpha:=\left[\alpha_{j}\right]_{j=1, \ldots, N} \in \mathbb{R}_{\geq 0}^{N}$ is a vector that describes the heterogeneous and unknown influence of each neuron on the electrode's recording. In the same way, we define the vector $\beta:=\left[\beta_{j}\right]_{j=1, \ldots, N} \in \mathbb{R}^{N}$, as the unknown gain of the electrical input on each neuron. The pair $(\alpha, \beta)$ thus defines the stimulation-registration setup. The dynamics of $N$ coupled oscillators under mean-field feedback then reads:

$\dot{z}_{i}=\left(\mathrm{i} \omega_{i}+\rho_{i}^{2}-\left|z_{i}\right|^{2}\right) z_{i}+\sum_{j=1}^{N} \kappa_{i j}\left(z_{j}-z_{i}\right)+\beta_{i} \sum_{j=1}^{N} \alpha_{j} \operatorname{Re}\left(z_{j}\right)$,

for all $i=1, \ldots, N$. Let us briefly compare the above model to existing ones. In (Rosenblum and Pikovsky, 2004; Tukhlina et al., 2007) the global dynamics of the network is modeled as a single Landau-Stuart oscillator, exploiting the fact that oscillators are synchronized. Hence that model is valid only near the synchronous state. On the contrary (3) is valid for both synchronized and desynchronized behaviors. In (Popovych et al., 2006) the authors use a population approach with all-to-all coupling that makes the results valid only for large number of oscillators. Our paper allows for general couplings and number of agents. Finally, we consider a real output as opposed to the complex output assumed in (Popovych et al., 2006).

In order to simplify the analysis, we make the assumption that each oscillator evolves with constant radius.

Hypothesis 1. (Constant radii). For all $i=1, \ldots, N$ there exists a constant $r_{i}>0$ such that the solution of (3) satisfies $\left|z_{i}(t)\right|=r_{i}$, for all $t \geq 0$.

This assumption is commonly made in synchronization studies (Acebrón et al., 2005; Aeyels and Rogge, 2004; Jadbabaie et al., 2004; Van Hemmen and Wreszinski, 1993; Brown et al., 2003; Kuramoto, 1984), and is justified by the normal hyperbolicity of the stable limit cycle of (1) that let the oscillation persist under external perturbations (cf. e.g. (Hoppensteadt and Izhikevich, 1997, Chapter 4.3)). Letting $z_{i}=r_{i} e^{\mathrm{i} \theta_{i}}$, which defines the phase $\theta_{i} \in \mathrm{T}^{1}$ of each oscillator, we get from Assumption 1 that $\dot{z}_{i}=\dot{r}_{i} e^{\mathrm{i} \theta_{i}}+$ $\mathrm{i} r_{i} \dot{\theta}_{i} e^{\mathrm{i} \theta_{i}}=\mathrm{i} r_{i} \dot{\theta}_{i} e^{\mathrm{i} \theta_{i}}$. Dividing each side of this equation by $r_{i} e^{\mathrm{i} \theta_{i}}$, and extracting the imaginary part of both sides, we get from (3) that

$\dot{\theta}_{i}=\omega_{i}+\sum_{j=1}^{N} \kappa_{i j} \frac{r_{j}}{r_{i}} \sin \left(\theta_{j}-\theta_{i}\right)-\beta_{i} \sin \left(\theta_{i}\right) \sum_{j=1}^{N} \alpha_{j} \frac{r_{j}}{r_{i}} \cos \left(\theta_{j}\right)$.

We can now use the trigonometric identity $\sin \theta_{i} \cos \theta_{j}=$ $\frac{1}{2} \sin \left(\theta_{j}+\theta_{i}\right)-\frac{1}{2} \sin \left(\theta_{j}-\theta_{i}\right)$ to derive

$$
\dot{\theta}_{i}=\omega_{i}+\sum_{j=1}^{N}\left(k_{i j}+\gamma_{i j}\right) \sin \left(\theta_{j}-\theta_{i}\right)-\sum_{j=1}^{N} \gamma_{i j} \sin \left(\theta_{j}+\theta_{i}\right),
$$

for all $i=1, \ldots, N$, where

$$
k=\left[k_{i j}\right]_{i, j=1, \ldots, N}:=\left[\kappa_{i j} \frac{r_{j}}{r_{i}}\right]_{i, j=1, \ldots, N} \in \mathbb{R}^{N \times N}
$$

is referred to as the coupling matrix, and

$$
\gamma=\left[\gamma_{i j}\right]_{i, j=1, \ldots, N}:=\left[\frac{\beta_{i}}{2} \frac{\alpha_{j} r_{j}}{r_{i}}\right]_{i, j=1, \ldots, N} \in \mathbb{R}^{N \times N}
$$

defines the feedback gain. We also define the modified coupling matrix, $\Gamma \in \mathbb{R}^{N \times N}$, as

$$
\Gamma:=\left[\Gamma_{i j}\right]_{i, j=1, \ldots, N}=\left[k_{i j}+\gamma_{i j}\right]_{i, j=1, \ldots, N} .
$$

We point out that the model (4) appears to be new in the literature and allows, by properly chosing the parameters 
$\alpha, \beta$ and $\kappa$, to encompass all kinds of interconnection topologies and recording-stimulation setups. We stress that the use of a nonzero feedback gains $\gamma$ breaks the $\mathrm{T}^{1}$ symmetry of the original Kuramoto system. That is, the invariance with respect to global phase shift no longer holds (Sepulchre et al., 2007).

\section{PHASE-LOCKED SOLUTIONS}

\subsection{Definition}

We start this section by formally defining the concept of phase-locking. Roughly speaking, a phase-locked solution can be interpreted as a fixed point of the incremental dynamics associated to (4). We distinguish solutions that exhibit collective oscillations (pathological case for DBS) from non-oscillating ones (neuronal inhibition).

Definition 1. (Phase-locked solution) A solution $\left\{\theta_{i}^{*}\right\}_{i=1, \ldots, N}$ of (4) is said to be phase-locked if it satisfies

$$
\dot{\theta}_{j}^{*}(t)-\dot{\theta}_{i}^{*}(t)=0, \quad \forall i, j=1, \ldots, N, \forall t \geq 0 .
$$

A phase-locked solution is oscillating if, in addition, $\dot{\theta}_{i}^{*}(t) \neq 0$, for almost all $t \geq 0$ and all $i=1, \ldots, N$.

In other words, for oscillating phase-locked solutions, the discharge rhythm is the same for each neuron, which corresponds to a synchronous (pathological) activity, while in the non oscillating case the neurons are in a quiescent non pathological state. The above definition of phase-locking corresponds to that of "Frequency (Huygens) Synchronization" (cf. e.g (Fradkov, 2007)). It is trivially equivalent to the existence of a matrix $\Delta:=\left[\Delta_{i j}\right]_{i, j=1, \ldots, N}$, such that

$$
\theta_{j}^{*}(t)-\theta_{i}^{*}(t)=\Delta_{i j}, \quad \forall i, j=1, \ldots, N, \forall t \geq 0,
$$

or to the existence of a measurable function $\Omega: \mathbb{R}_{\geq 0} \rightarrow \mathbb{R}$ such that, for each $i=1, \ldots, N$,

$$
\theta_{i}^{*}(t)=\int_{0}^{t} \Omega(s) d s+\theta_{i}^{*}(0), \quad \forall t \geq 0,
$$

where $\Omega$ is the instantaneous collective frequency of oscillation, that is $\dot{\theta}_{i}^{*}(t)=\Omega(t)$ for all $i=1, \ldots, N$. In case of oscillating phase-locking, $\Omega(t) \neq 0$ for almost all $t \geq 0$. In the Kuramoto system without mean-field feedback, the oscillating and non-oscillating cases are equivalent due to the $T^{1}$ symmetry, which guarantees invariance to a common phase drift such as a nonzero mean natural frequency (Sepulchre et al., 2007, Eq. (8)). We note that a simple sufficient condition to avoid oscillator death (i.e. nonoscillating phase-locking) is given by

$\max _{i=1, \ldots, N}\left|\omega_{i}\right|>\max _{i=1, \ldots, N} \sum_{j=1, j \neq i}^{N}\left|k_{i j}+\gamma_{i j}\right|+\max _{i=1, \ldots, N} \sum_{j=1}^{N}\left|\gamma_{i j}\right|$, meaning that at least one natural frequency is sufficiently large with respect to the coupling and feedback gain. This condition ensures that the phase dynamics (4) does not have fixed points.

\subsection{Existence of oscillating phase-locking}

We now present a general result on phase-locking under mean-field feedback. Its proof is given in Section 4.3. We recall that the expression "for almost all" defines sets whose complement has zero Lebesgue measure in the interested space.
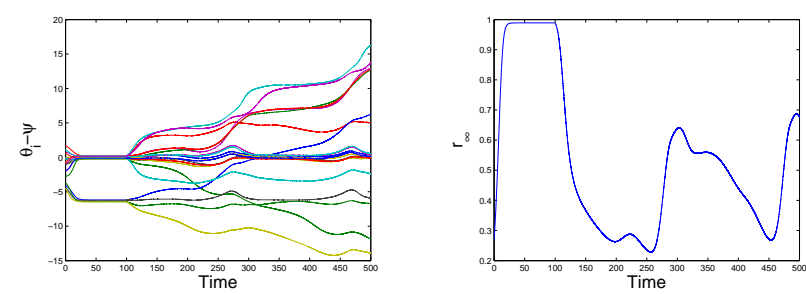

Fig. 1. Large feedback gain, full desynchronization.
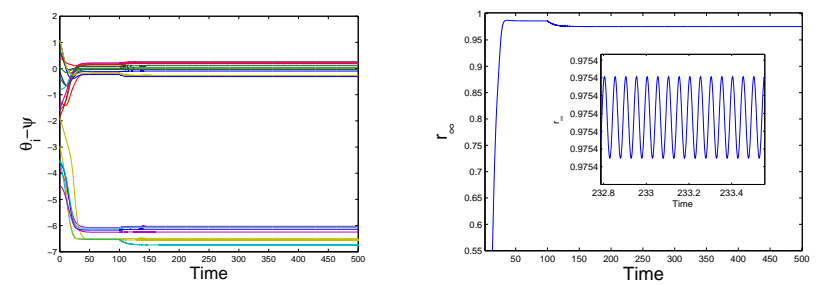

Fig. 2. Small feedback gain, practical phase-locking.

Theorem 2. (No phase-locked solution). Given $N \geq 2$, for almost all natural frequencies $\omega \in \mathbb{R}^{N}$, for almost all interconnection matrices $k \in \mathbb{R}^{N \times N}$, and for almost all feedback gains $\gamma \in \mathbb{R}^{N \times N}$, system (4) admits no oscillating phase-locked solution.

Theorem 2 states that, for a generic ${ }^{1}$ neuronal interconnection, the use of a proportional mean-field feedback prevents the oscillators to all evolve at the exact same frequency. Generically, under mean-field feedback, only two situations may therefore occur: either no phase-locking or no oscillations. This result therefore constitutes a promising feature of mean-field feedback DBS.

On the one hand, the strength of Theorem 2 stands in the generality of its assumptions: it holds for generic interconnections between neurons, including negative weights corresponding to inhibitory synapses, and does not require any knowledge neither on the contribution $\alpha_{j}$ of each neuron on the overall measurement nor on the intensity $\beta_{j}$ of the stimulation on each neuron.

On the other hand, the disappearance of the phase-locked state does not imply the end of the pathological behavior. Indeed, while Theorem 2 states that the perfectly synchronized behavior is not compatible with mean-field feedback, it does not exclude the possibility that the system shows some kind of "practical" phase-locking, such as solutions whose mean behavior is near to that of a phase-locked one, but with small oscillations around it. This problem is addressed in (Franci et al., 2010, Section 4).

Numerical simulations illustrate these two features. The plots of (a) the phase differences with respect to their mean $\psi$ and (b) the order parameter $r_{\infty}$, given by $r_{\infty} e^{i \psi}:=$ $\frac{1}{N} \sum_{j=1}^{N} e^{\mathrm{i} \theta_{j}}$, are provided for a large (Fig. 1) and a small (Fig. 2) feedback gain. Mean-field feedback is applied at $t=100$. While full desynchronization is achieved for the large feedback gain, practical phase-locking is observed in case of a too small feedback.

1 A counter-example to Theorem 2 that shows its generic nature can be found in the on-line extended version of (Franci et al., 2010). 


\subsection{Characterization of phase-locking}

The proof of Theorem 2 (Section 4.3) is based on two main steps, which are presented here as Lemmas 3 and 4.

Lemma 3 states that the problem of finding a phaselocked solution can be reduced to solving a set of nonlinear algebraic equations in terms of the phase differences $\Delta$ and the collective frequency of oscillation $\Omega$. Its proof is provided in Section 4.1.

Lemma 3. (Fixed-point equation). Given $N \geq 2$, for all initial conditions $\theta^{*}(0) \in \mathbb{R}^{N}$, all natural frequencies $\omega \in \mathbb{R}^{N}$, all coupling matrices $k \in \mathbb{R}^{N \times N}$, and all feedback gains $\gamma \in \mathbb{R}^{N \times N}$, if system (4) admits an oscillating phaselocked solution starting in $\theta^{*}(0)$ with phase differences $\Delta$ and collective frequency of oscillation $\Omega$ satisfying (9)-(10), then, for all $1 \leq i<j \leq N$, it holds that

$$
\begin{gathered}
\omega_{j}-\omega_{i}+\sum_{l=1}^{N}\left[\left(k_{j l}+\gamma_{j l}\right) \sin \left(\Delta_{j l}\right)-\left(k_{i l}+\gamma_{i l}\right) \sin \left(\Delta_{i l}\right)\right]=0 \\
\sum_{l=1}^{N}\left[\gamma_{j l} \sin \left(2 \int_{0}^{t} \Omega(s) d s+\Delta_{j l}+2 \theta_{j}^{*}(0)\right)\right. \\
\left.-\gamma_{i l} \sin \left(2 \int_{0}^{t} \Omega(s) d s+\Delta_{i l}+2 \theta_{i}^{*}(0)\right)\right]=0
\end{gathered}
$$

While this fact is trivial for the Kuramoto system without inputs (i.e. $\gamma=0$ ), its generalization to the presence of mean-field feedback is not straightforward. The first set of equations (11a) can be seen as the "standard" fixed point equation for a Kuramoto system with natural frequencies $\omega$ and coupling matrix $\Gamma=k+\gamma$. It may or may not lead to the existence of a phase-locked solution (see (Jadbabaie et al., 2004) for necessary and sufficient conditions). The second set of equations (11b) is linked to the action of the mean-field feedback. It trivially holds if the feedback gain $\gamma$ is zero.

Intuitively, we can expect that if the frequency of the collective oscillation $\Omega$ is not zero then (11b) admits no solution for any $\gamma \neq 0$. The second main step in the proof of Theorem 2 confirms that indeed, if (11) admits a solution $(\Delta, \Omega)$, then $\Delta$ is fully determined by the "standard" part (11a) of this fixed point equation. In particular the following lemma states that, around almost any solution of (11a), the phase differences that define a phase-locked configuration $\Delta$ can be locally expressed as a smooth function of the natural frequencies $\omega$ and of the interconnection matrix $\Gamma$.

Lemma 4. (Invertibility of the Kuramoto fixed point equation). Given $N \geq 2$, there exists a set $\mathcal{N} \subset \mathbb{R}^{N} \times \mathbb{R}^{N \times N}$, and a set $\mathcal{N}_{0} \subset \mathcal{N}$ satifying $\mu\left(\mathcal{N}_{0}\right)=0$, such that (11a) with natural frequencies $\omega^{*} \in \mathbb{R}^{N}$ and modified interconnection matrix $\Gamma^{*}:=k^{*}+\gamma^{*} \in \mathbb{R}^{N \times N}$ admits a solution $\Delta^{*} \in \mathbb{R}^{N \times N}$ if and only if $\left(\omega^{*}, \Gamma^{*}\right) \in \mathcal{N}$. Moreover, for all $\left(\omega^{*}, \Gamma^{*}\right) \in \mathcal{N} \backslash \mathcal{N}_{0}$, there exists a neighborhood $U$ of $\left(\omega^{*}, \Gamma^{*}\right)$, a neighborhood $W$ of $\Delta^{*}$, and a smooth function $f: U \rightarrow W$, such that, for all $(\omega, \Gamma) \in U$, $(\omega, \Gamma, \Delta:=f(\omega, \Gamma))$ is the unique solution of (11a) in $U \times W$.

The rest of the document aims at proving these two lemmas and establish Theorem 2.

\section{PROOFS}

4.1 Proof of Lemma 3

From (9)-(10), phase-locked solutions of (4) satisfy, for all $t \geq 0$ and all $i, j=1, \ldots, N, \theta_{j}^{*}(t)+\theta_{i}^{*}(t)=\theta_{j}^{*}(t)-\theta_{i}^{*}(t)+$ $2 \theta_{i}^{*}(t)=2 \Lambda_{\Omega}(t)+\Delta_{i j}+2 \theta_{i}^{*}(0)$, where $\Lambda_{\Omega}(t):=\int_{0}^{t} \Omega(s) d s$, for all $t \geq 0$. In view of (9) and (10), and noting that the fixed point equation equation is trivial if $i=j$ and that, due to the antisymmetric dependence of (11) on $i$ and $j$, finding a solution for some $i=i^{*}$ and $j=j^{*}$ gives a solution also for $i=j^{*}$ and $j=i^{*}$, finding a phase-locked solution is equivalent to solving the set of equations

$$
\begin{aligned}
\omega_{j}-\omega_{i}+ & \sum_{l=1}^{N}\left[\left(k_{j l}+\gamma_{j l}\right) \sin \left(\Delta_{j l}\right)-\left(k_{i l}+\gamma_{i l}\right) \sin \left(\Delta_{i l}\right)\right]- \\
& \sum_{l=1}^{N}\left[\gamma_{j l} \sin \left(2 \Lambda_{\Omega}(t)+\Delta_{j l}+2 \theta_{j}^{*}(0)\right)-\right. \\
& \left.\gamma_{i l} \sin \left(2 \Lambda_{\Omega}(t)+\Delta_{i l}+2 \theta_{i}^{*}(0)\right)\right]=0
\end{aligned}
$$

for all $t \geq 0$ and all $1 \leq i<j \leq N$, in terms of $\Delta$ and $\Omega$. Note that the first line of the last equation is constant, that is there exists a set of constants $\left\{c_{i j}\right\}_{i, j=1, \ldots, N, i<j}$ such that

$$
\omega_{j}-\omega_{i}+\sum_{l=1}^{N}\left[\left(k_{j l}+\gamma_{j l}\right) \sin \left(\Delta_{j l}\right)-\left(k_{i l}+\gamma_{i l}\right) \sin \left(\Delta_{i l}\right)\right]=c_{i j},
$$

(12) then reads

$$
\begin{aligned}
& \sum_{l=1}^{N}\left[\gamma_{j l} \sin \left(2 \Lambda_{\Omega}(t)+\Delta_{j l}+2 \theta_{j}^{*}(0)\right)-\right. \\
& \left.\gamma_{i l} \sin \left(2 \Lambda_{\Omega}(t)+\Delta_{i l}+2 \theta_{i}^{*}(0)\right)\right]=c_{i j},
\end{aligned}
$$

for all $t \geq 0$ and all $1 \leq i<j \leq N$. Hence, if a phaselocked solution exists, then there must exists a solution to the set of equations (13) and (14) in terms of $\Delta, \Omega$ and $c:=\left\{c_{i j}\right\}_{i, j=1, \ldots, N, i<j}$. By differentiating (14) with respect to time, one gets, for all $t \geq 0$ and all $1 \leq i<j \leq N$,

$$
\begin{gathered}
2 \Omega(t) \sum_{l=1}^{N}\left[\gamma_{j l} \cos \left(2 \Lambda_{\Omega}(t)+\Delta_{j l}+2 \theta_{j}^{*}(0)\right)\right. \\
\left.-\gamma_{i l} \cos \left(2 \Lambda_{\Omega}(t)+\Delta_{i l}+2 \theta_{i}^{*}(0)\right)\right]=0 .
\end{gathered}
$$

Since we consider an oscillating phase-locked solution, $\Omega$ is a non identically zero continuous function. Hence, there exists an open interval $(\underline{t}, \bar{t}), 0 \leq \underline{t}<\bar{t}$, such that $\Omega(t) \neq 0$ for all $t \in(\underline{t}, \bar{t})$. Hence $(15)$ implies that

$$
\begin{gathered}
\sum_{l=1}^{N}\left[\gamma_{j l} \cos \left(2 \Lambda_{\Omega}(t)+\Delta_{j l}+2 \theta_{j}^{*}(0)\right)\right. \\
\left.-\gamma_{i l} \cos \left(2 \Lambda_{\Omega}(t)+\Delta_{i l}+2 \theta_{i}^{*}(0)\right)\right]=0,
\end{gathered}
$$

for all $t \in(\underline{t}, \bar{t})$. By differentiating (16) with respect to time and considering once again that $\Omega(t) \neq 0$ for all $t \in(\underline{t}, \bar{t})$, one gets that (14) holds true for all $t \in(\underline{t}, \bar{t})$, and for all $1 \leq i<j \leq N . c_{i j}$ being a constant this implies that, for all $t \geq 0, c_{i j}=0$, for all $1 \leq i<j \leq N$.

\subsection{Proof of Lemma 4}

For notational purposes, define $y_{i}:=\Delta_{i N}, i=1, \ldots, N-1$, and $y:=\left[y_{i}\right]_{i=1, \ldots, N-1}$. Since it holds that $\Delta_{m n}=y_{n}-y_{m}$, for all $m, n=1, \ldots, N$, we can express all the phase differences in (11a) in terms of the components of $y$. 
Analogously, since $\dot{\theta}_{n}-\dot{\theta}_{m}=\dot{\theta}_{n}-\dot{\theta}_{N}-\left(\dot{\theta}_{m}-\dot{\theta}_{N}\right)$, it suffices to find a solution to the set of equation in (11a), relative to the pairs of index $(i, N), i=1, \ldots, N-1$. For the vectors $y$, we use the suffix ${ }^{*}$ when it refers to $\Delta^{*}$. Define, for $i=1, \ldots, N-1$,

$$
\begin{aligned}
& F_{i}(\omega, \Gamma, y):=\omega_{N}-\omega_{i}- \\
& \sum_{k=1}^{N-1}\left[\Gamma_{i k} \sin \left(y_{k}-y_{i}\right)-\Gamma_{N k} \sin y_{k}\right]-\Gamma_{i N} \sin y_{i} .
\end{aligned}
$$

With this notation, the equations in (11a) relative to the pairs of index $(i, N), i=1, \ldots, N-1$, can be written as

$$
F(\omega, \Gamma, y)=0 \text {. }
$$

In order to solve (17)in the form $y=f(\Gamma, \omega)$ through the implicit function theorem, we have to show that the matrix

$$
J(\Gamma, y):=\left[\frac{\partial F_{i}}{\partial y_{j}}(\Gamma, y)\right]_{i, j=1, \ldots, N-1},
$$

has full rank on the solutions of (17). Define, for $y \in \mathbb{R}^{N-1}$,

$$
S(y):=\left\{\Gamma \in \mathbb{R}^{N \times N}: \operatorname{det} J(\Gamma, y)=0\right\} .
$$

Since det $J(\Gamma, y)$ is a non-identically zero analytical function (for example it is non-zero for $y=0$ and $\Gamma_{i j}=1$, for all $i, j=1, \ldots, N)$, it is zero only on sets of zero measure (Krantz and Parks, 2002, Chapter 4). In particular $\mu(S(y))=0$, for all $y \in \mathbb{R}^{N-1}$. Let the sets $\mathcal{N}$ and $\mathcal{N}_{0}$ be defined as

$$
\begin{aligned}
\mathcal{N}:=\left\{(\omega, \Gamma) \in \mathbb{R}^{N} \times \mathbb{R}^{N \times N}:\right. & \\
& \left.\exists y \in \mathbb{R}^{N-1}: F(\omega, \Gamma, y)=0\right\}
\end{aligned}
$$

and

$$
\begin{aligned}
\mathcal{N}_{0} & :=\left\{(\omega, \Gamma) \in \mathbb{R}^{N} \times \mathbb{R}^{N \times N}:\right. \\
& \left.\exists y \in \mathbb{R}^{N-1}: F(\omega, \Gamma, y)=0, \Gamma \in S(y)\right\},
\end{aligned}
$$

that is $\mathcal{N}$ contains all the natural frequencies and modified interconnection matrices that admit a solution to (11a), and $\mathcal{N}_{0}$ all the natural frequencies and modified interconnection matrices that admit a solution to (11a) such that (18) is singular. The next claim, proved in the on-line available extended version of Franci et al. (2010), shows that $\mathcal{N}_{0}$ is of zero Lebesgue measure.

Claim 1. Let $\mathcal{N}_{0}$ be defined as in (21). Then $\mu\left(\mathcal{N}_{0}\right)=0$.

The lemma then follows directly from the implicit function theorem. Indeed, given any point $\left(\omega^{*}, \Gamma^{*}\right) \in \mathcal{N} \backslash \mathcal{N}_{0}$, since $\Gamma^{*} \notin S\left(y^{*}\right)$, the matrix $J\left(\Gamma^{*}, y^{*}\right)$, defined in (18), is invertible, and the existence of the neighborhoods $U$ and $W$, and of the function $f$ with the properties of the statement of the lemma follows directly from the implicit function theorem (Lee, 2006, Theorem 7.9).

\subsection{Proof of Theorem 2}

The proof consists in explicitly constructing a zero Lebesgue measure set of natural frequencies and coupling and feedback gains, out of which the system of equations (11) admits no solutions. The theorem then follows from Lemma 3.

Since the interconnection matrix $k$, the modified interconnection matrix $\Gamma$, and the feedback gain $\gamma$ are linked by the linear relation $k=\Gamma-\gamma$, we can independently fix $\Gamma$ and $\gamma$, and set $k$ accordingly. For all $\omega \in \mathbb{R}$, let

$$
\mathcal{M}_{0}(\omega):=\left\{\Gamma \in \mathbb{R}^{N \times N}:(\omega, \Gamma) \in \mathcal{N}_{0}\right\}
$$

where $\mathcal{N}_{0} \subset \mathbb{R}^{N} \times \mathbb{R}^{N \times N}$ is defined in the statement of Lemma 4 and has zero Lebesgue measure. Let

$$
\mathcal{S}_{0}:=\left\{\omega \in \mathbb{R}^{N}: \mu\left(\mathcal{M}_{0}(\omega)\right)>0\right\} .
$$

If $\mu\left(\mathcal{S}_{0}\right)>0$, then $\mu\left(\mathcal{N}_{0}\right)=\int_{\mathcal{S}_{0}} \mu\left(\mathcal{M}_{0}(\omega)\right) d \omega>0$, which contradicts Lemma 4. Hence $\mu\left(\mathcal{S}_{0}\right)=0$.

Consider any $\omega \in \mathbb{R}^{N} \backslash \mathcal{S}_{0}$ and any $\Gamma \in \mathbb{R}^{N \times N} \backslash \mathcal{M}_{0}(\omega)$. In view of what precedes, this constitutes a generic choice or $\omega$ and $\Gamma$ and it holds that $(\omega, \Gamma) \notin \mathcal{N}_{0}$. Suppose that there exists an oscillating phase-locked solution starting in $\theta^{*}(0)$, with phase differences $\Delta$ and collective frequency of oscillation $\Omega$. From Lemma 3 , a necessary condition for the existence of an oscillating phase locked solution $\theta^{*}$ is that $(\omega, \Gamma, \Delta)$ is a solution of (11a). From Lemma 4, $(\omega, \Gamma) \in \mathcal{N}$. Since $(\omega, \Gamma) \in \mathcal{N} \backslash \mathcal{N}_{0}$, Lemma 4 guarantees that the phase differences $\Delta$ of $\theta^{*}$ can locally be uniquely expressed in the form $\Delta=f(\omega, \Gamma)$, for some smooth function $f: \mathbb{R}^{N} \times \mathbb{R}^{N \times N} \rightarrow \mathbb{R}^{N \times N}$. In particular $\Delta$ does not depend on the feedback gain $\gamma$. Consider now the line of $(11 \mathrm{~b})$ relative to the pair of indices $(1,2)$ :

$$
\begin{gathered}
\sum_{i=1}^{N}\left[\gamma_{1 i} \sin \left(\Lambda_{\Omega}(t)+\Delta_{1 i}+2 \theta_{1}^{*}(0)\right)\right. \\
\left.-\gamma_{2 i} \sin \left(\Lambda_{\Omega}(t)+\Delta_{2 i}+2 \theta_{2}^{*}(0)\right)\right]=0, \forall t \geq 0,
\end{gathered}
$$

where $\Lambda_{\Omega}(t):=2 \int_{0}^{t} \Omega(s) d s$, for all $t \geq 0$. Using the identity $\sin (a+b)=\sin a \cos b+\cos a \sin b$ and defining

$$
\begin{aligned}
& \Sigma_{1}:=\sum_{i=1}^{N} \gamma_{1 i} \cos \left(\Delta_{1 i}+2 \theta_{1}^{*}(0)\right)-\gamma_{2 i} \cos \left(\Delta_{2 i}+2 \theta_{2}^{*}(0)\right) \\
& \Sigma_{2}:=\sum_{i=1}^{N} \gamma_{1 i} \sin \left(\Delta_{1 i}+2 \theta_{1}^{*}(0)\right)-\gamma_{2 i} \sin \left(\Delta_{2 i}+2 \theta_{2}^{*}(0)\right)
\end{aligned}
$$

Equation (24) reads $\Sigma_{1} \sin \Lambda_{\Omega}(t)-\Sigma_{2} \cos \Lambda_{\Omega}(t)=0$. Since $\sin \Lambda_{\Omega}(0)=0$ and $\cos \Lambda_{\Omega}(0)=1, \Sigma_{2}$ has to be zero. Moreover, since the phase-locked solution $\theta^{*}$ is oscillating, there exists $t>0$ such that $\sin \Lambda_{\Omega}(t) \neq 0$. Hence, $\Sigma_{1}=0$ as well. Define $b_{1}, b_{2} \in \mathbb{R}^{2 N}$ as

$$
\begin{aligned}
b_{1}(\Delta):= & {\left[\left[\cos \left(\Delta_{1 i}+2 \theta_{1}^{*}(0)\right)\right]_{i=1, \ldots, N}^{T},\right.} \\
& \left.-\left[\cos \left(\Delta_{2 i}+2 \theta_{2}^{*}(0)\right)\right]_{i=1, \ldots, N}^{T}\right]^{T}, \\
b_{2}(\Delta):= & {\left[\left[\sin \left(\Delta_{1 i}+2 \theta_{1}^{*}(0)\right)\right]_{i=1, \ldots, N}^{T},\right.} \\
& \left.-\left[\sin \left(\Delta_{2 i}+2 \theta_{2}^{*}(0)\right)\right]_{i=1, \ldots, N}^{T}\right]^{T} .
\end{aligned}
$$

Note that $b_{1}$ and $b_{2}$ depend only on $\Delta=f(\omega, \Gamma)$ and on the initial conditions. They do not depend on $\gamma$. Hence, by defining $\tilde{\gamma}:=\left(\left[\gamma_{2 i}\right]_{i=1, \ldots, N}^{T},\left[\gamma_{1 i}\right]_{i=1, \ldots, N}^{T}\right)^{T} \in \mathbb{R}^{2 N}$, the condition $\Sigma_{1}=\Sigma_{2}=0$ can be re-written as $\tilde{\gamma}^{T} b_{1}=$ $\tilde{\gamma}^{T} b_{2}=0$ or, equivalently,

Let

$$
\tilde{\gamma} \in b_{1}(f(\omega, \Gamma))^{\perp} \cap b_{2}(f(\omega, \Gamma))^{\perp} .
$$

$$
\mathcal{L}_{0}(\omega, \Gamma):=b_{1}(f(\omega, \Gamma))^{\perp} \cap b_{2}(f(\omega, \Gamma))^{\perp} \subset \mathbb{R}^{2 N} .
$$

Noticing that $b_{1}, b_{2}$ can not be both zero, $\mu\left(\mathcal{L}_{0}(\omega, \Gamma)\right)=0$ for all $\omega, \Gamma \in \mathbb{R} \times \mathbb{R}^{N \times N}$. Recalling (27), for all $\omega \in \mathbb{R}^{N} \backslash \mathcal{S}_{0}$, for all $\Gamma \in \mathbb{R}^{N \times N} \backslash \mathcal{M}_{0}(\omega)$, and for all $\gamma \in \mathbb{R}^{N \times N} \backslash \mathcal{L}_{0}(\omega, \Gamma)$, system (4) admits no oscillating phase-locked solution, where $\mathcal{M}_{0}, \mathcal{S}_{0}$, and $\mathcal{L}_{0}$ are defined in (22),(23), and (28), respectively, and are all of zero Lebesgue measure. The theorem is proved by noticing that, given $\omega \in \mathbb{R} \backslash \mathcal{S}_{0}$, for 
any $\Gamma \in \mathbb{R}^{N \times N} \backslash \mathcal{M}_{0}(\omega)$, the set $\left\{\gamma \in \mathbb{R}^{N \times N}: \gamma=\Gamma-\right.$ $\left.k, k \in \mathbb{R}^{N \times N}\right\}=\mathbb{R}^{N \times N}$, that is, given any $\Gamma \in \mathbb{R}^{N \times N}$ $\mathcal{M}_{0}(\omega), \gamma \notin \mathcal{L}_{0}(\omega, \Gamma)$ for almost all $k$.

\section{REFERENCES}

Acebrón, J.A., Bonilla, L.L., Vicente, C.J.P., Ritort, F., and Spigler, R. (2005). The Kuramoto model: A simple paradigm for synchronization phenomena. Reviews of modern physics, $77,137-185$.

Aeyels, D. and Rogge, J.A. (2004). Existence of partial entrainment and stability of phase locking behavior of coupled oscillators. Progress of Theoretical Physics, 112(6), 921-942.

Alberts, W.W., Wright, E.J., and Feistein, B. (1969). Cortical potentials and Parkinsonian tremor. Nature, 221, 670-672.

Benabid, A.L., Pollak, P., Gervason, C., Hoffmann, D., Gao, D.M., Hommel, M., Perret, J.E., and de Rougemont, J. (1991). Long-term suppression of tremor by chronic stimulation of the ventral intermediate thalamic nucleus. The Lancet, 337, 403-406.

Brown, E., Holmes, P., and Moehlis, J. (2003). Globally coupled oscillator networks. In K. Sreenivasan, E. Kaplan, and J. Marsden (eds.), Perspectives and Problems in Nonlinear Science: A Celebratory Volume in Honor of Larry Sirovich. New York.

Chopra, N. and Spong, M.W. (2009). On exponential synchronization of Kuramoto oscillators. IEEE Trans. on Automat. Contr., 54(2), 353-357.

Fradkov, A.L. (2007). Cybernetical Physics. From Control of Chaos to Quantum Control. Springer: Complexity. Springer-Verlag, Berlin Heidelberg.

Franci, A., Chaillet, A., and Pasillas-Lépine, W. (2010). Existence and robustness of phase-locking in coupled Kuramoto oscillators under mean-field feedback. To appear in: Automatica - Special Issue on Biology Systems. Extended version available at: http://hal.archives-ouvertes.fr/hal00526066/.

Hammond, C., Ammari, R., Bioulac, B., and Garcia, L. (2008). Latest view on the mechanism of action of deep brain stimulation. Movement Disorders, 23(15), 21112121.

Hoppensteadt, F.C. and Izhikevich, E.M. (1997). Weakly connected neural networks, volume 126 of Applied Mathematical Sciences. Springer-Verlag, New York.

Izhikevich, E.M. (2007). Dynamical Systems in Neuroscience: The Geometry of Excitability and Bursting. MIT Press, Cambridge, Massachusetts, London, England.

Jadbabaie, A., Motee, N., and Barahona, M. (2004). On the stability of the Kuramoto model of coupled nonlinear oscillators. Proc. American Control Conf., 4296-4301.

Krantz, S. and Parks, H. (2002). A primer of real analytic functions. Birkhäuser, Boston - Basel - Berlin.

Kumar, R., Lozano, A.M., Sime, E., and Lang, A.E. (2003). Long-term follow-up of thalamic deep brain stimulation for essential and Parkinsonian tremor. Neurology, 61, 1601-1604.

Kuramoto, Y. (1984). Chemical Oscillations, Waves, and Turbulence. Springer, Berlin.

Lee, J. (2006). Introduction to smooth manifolds. Graduate Texts in Mathematics. Springer-Verlag, Berlin, Germany.
Nijmeijer, H. and Rodriguez-Angeles, A. (2003). Synchronization of Mechanical Systems, volume 46. World Scientific Series on Nonlinear Science, Series A.

Olfati-Saber, R. and Murray, R.M. (2004). Consensus problems in networks of agents with switching topology and time-delays. IEEE Trans. on Automat. Contr., 49(9), 1520-1533.

Pikovsky, A., Rosenblum, M., and Kurths, J. (2001). Synchronization: A Universal Concept in Nonlinear Sciences. Cambridge Nonlinear Science Series, Cambridge, United Kingdom.

Popovych, O.V., Hauptmann, C., and Tass, P.A. (2006). Desynchronization and decoupling of interacting oscillators by nonlinear delayed feedback. Internat. J. Bifur. Chaos, 16(7), 1977-1987. doi: 10.1142/S0218127406015830.

Rodriguez-Oroz, M.C. and et al. (2005). Bilateral deep brain stimulation in Parkinson's disease: a multicentre study with 4 years follow-up. Brain, 128, 2240-2249.

Rosenblum, M. and Pikovsky, A. (2004). Delayed feedback control of collective synchrony: an approach to suppression of pathological brain rhythms. Phys. Rev. E, 70(4), 041904. doi:10.1103/PhysRevE.70.041904.

Sarlette, A. (2009). Geometry and Symmetries in Coordination Control. Ph.D. thesis, University of Liège, (B).

Sarma, S.V., Cheng, M., Williams, Z., Hu, R., Eskandar, E., and Brown, E.N. (2010). Comparing healthy and Parkinsonian neuronal activity in sub-thalamic nucleus using point process models. IEEE Trans Biomed Eng., 57(6), 1297-1305.

Scardovi, L., Sarlette, A., and Sepulchre, R. (2007). Synchronization and balancing on the N-torus. Syst. \&6 Contr. Letters, 56(5), 335-341.

Sepulchre, R., Paley, D., and Leonard, N.E. (2008). Stabilization of planar collective motion with limited communication. IEEE Trans. on Automat. Contr., 53(3), 706-719.

Sepulchre, R., Paley, D.A., and Leonard, N.E. (2007). Stabilization of planar collective motion: All-to-all communication. IEEE Trans. on Automat. Contr., 52(5), 811-824.

Strogatz, S.H. (2000). From Kuramoto to Crawford: Exploring the onset of synchronization in population of coupled oscillators. Physica D, 143, 1-20.

Tukhlina, N., Rosenblum, M., Pikovsky, A., and Kurths, J. (2007). Feedback suppression of neural synchrony by vanishing stimulation. Physical Review E, 75(1), 011918.

Van Hemmen, J.L. and Wreszinski, W.F. (1993). Lyapunov function for the Kuramoto model on nonlinearly coupled oscillators. Jour. of Statistical Physics, 72, 145166.

Volkmann, J., Joliot, M., Mogilner, A., Ioannides, A.A., Lado, F., Fazzini, E., Ribary, U., and Llinás, R. (1996). Central motor loop oscillations in Parkinsonian resting tremor revealed by magnetoencephalography. Neurology, 46, 1359-1370.

Winfree, A.T. (1980). The Geometry of Biological Times. Springer, New-York. 